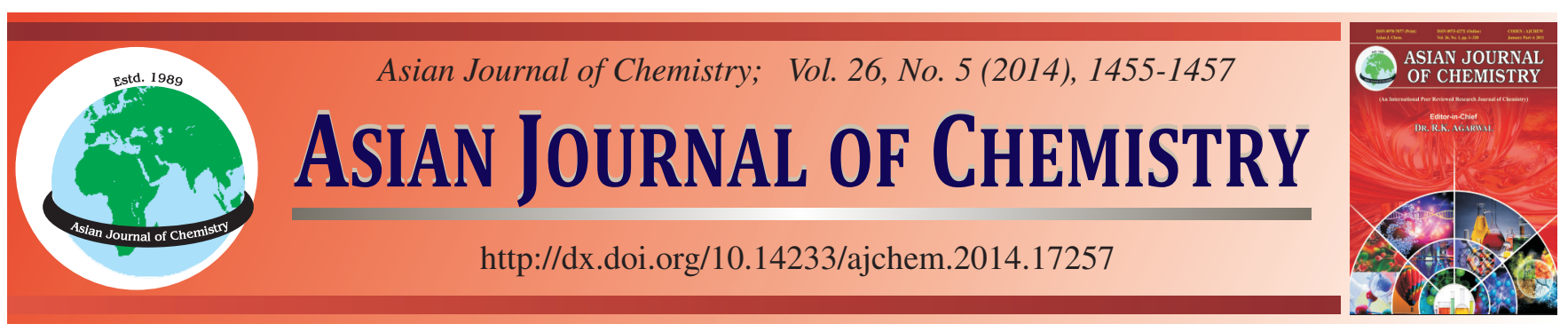

\title{
Electronic and Transport Properties Depending on the Edge Termination in Zigzag Graphene Nanoribbons ${ }^{\dagger}$
}

\author{
JiNG HUANG* and RONG XIE
}

School of Materials and Chemical Engineering, Anhui Jianzhu University, Hefei, P.R. China

*Corresponding author: E-mail: jhuang@ustc.edu.cn

We reported here the first-principle studies on the electronic and transport properties of the zigzag graphene nanoribbons which are terminated by the different edge functional groups including the oxygen atoms as well as the imine groups. The calculated results indicated that the electronic structures of zigzag graphene nanoribbons are significantly determined by the concentration of the chemical edge terminations. The obtained band structures presented the obviously variations from the spin-restricted metal characteristics, the semi-conductive behaviors to the spin-polarized states with the decrease of the concentration for the substituted groups in zigzag graphene nanoribbons. Those results lead to the effectively controlling to the low-bias transport properties of the junctions based on these kinds of zigzag graphene nanoribbons.

Keywords: Zigzag graphene, Electronic structure, Transport properties, DFT.

\section{INTRODUCTION}

Graphene nanoribbons (GNRs) are the promising candidate materials for building the novel molecular devices because of their inherent advantages such as more straightforward fabricated processes and better tailing. As we know, with the different edge-structure, the graphene nanoribbons can present two different geometrical structures: the zigzag and the armchair graphene nanoribbons. Previous theoretical and experimental studies show that the physical and chemical properties of graphene nanoribbons are depended on the different chemical functional groups since there present relatively high electron density at the edge and those particular electronic states are located around the Fermi level. Recently, many researched attentions have been focused on the tuning the electronic and transport properties due to the varied edge chemical atoms and groups.

It is well known that ground states of zigzag graphene nanoribbons are the spin degenerate in all bands when they are terminated by the hydrogen atoms on both sides ${ }^{1}$. Theoretical calculated results demonstrated that the half-metallic states can be obtained through the presence of the transverse electric filed based on the different width ${ }^{2-4}$. Of cause, excepting for the external transverse field, people can also realize the tuning of the electronic structures via the edge chemical functional groups, such as the doping and the modifying of the edge hydrogen atoms ${ }^{2-4}$. Gunlycke et al. ${ }^{1}$ found that the band structures near the Fermi level have the dramatically different characteristics terminated with the varied groups. So it's possible that people can design various electronic devices according to the edge chemical groups.

Unfortunately, previous works are focused on the substitution of all hydrogen edge atoms. Another interesting topic is to investigate the influence of the different substituted concentration on the electronic and transport properties. In this paper, we theoretically investigated the electronic and transport properties of zigzag graphene nanoribbons using the density functional theory (DFT) combined with the non-equilibrium Green's functions. The calculated results indicated that zigzag graphene nanoribbons present the varied ground states, including the metallic and the semi-conductor with spin-restricted states and the spin-resolved states due to the different concentration of the substituted edge groups. These predictions demonstrated that zigzag graphene nanoribbons with the different terminated chemical groups will hold great promising in molecular electronic and spintronics applications. 


\section{THEORETICAL CALCULATIONS}

Calculated method and model: The geometric, electronic and transport properties are calculated by the spin-resolved local-density functional theory combined with non-equilibrium Green's functions technology which are implemented in the Spanish Initiative for Electronic Simulations with Thousands of Atoms (SIESTA-3.0) package ${ }^{5,6}$. In our calculations, CeperleyAlder LSDA is used to describe the exchange and correlation energy. A double-zeta plus polarization (DZP) basis sets are used for all elements including the carbon, nitrogen, oxygen and hydrogen atoms and an energy cutoff of $150 \mathrm{Ry}$ for the grid integration is set to present the accurate charge density ${ }^{7}$.

Our proposed zigzag graphene nanoribbons are shown in Fig. 1, which present the different concentration of the substituted groups. In the schematic, the blue balls which have been signed with " $\mathrm{X}$ " denoted the position of the oxygen atoms (the imine groups). The black and green atoms indicate the carbon and hydrogen atoms, respectively. The optimized carbon-carbon distance is $1.42 \AA$ which is the typical theoretical values. While, the distance between the carbon and oxygen atoms (carbon-nitrogen) varied from 1.30-1.40 ̊ due to the different concentration of substituted groups.

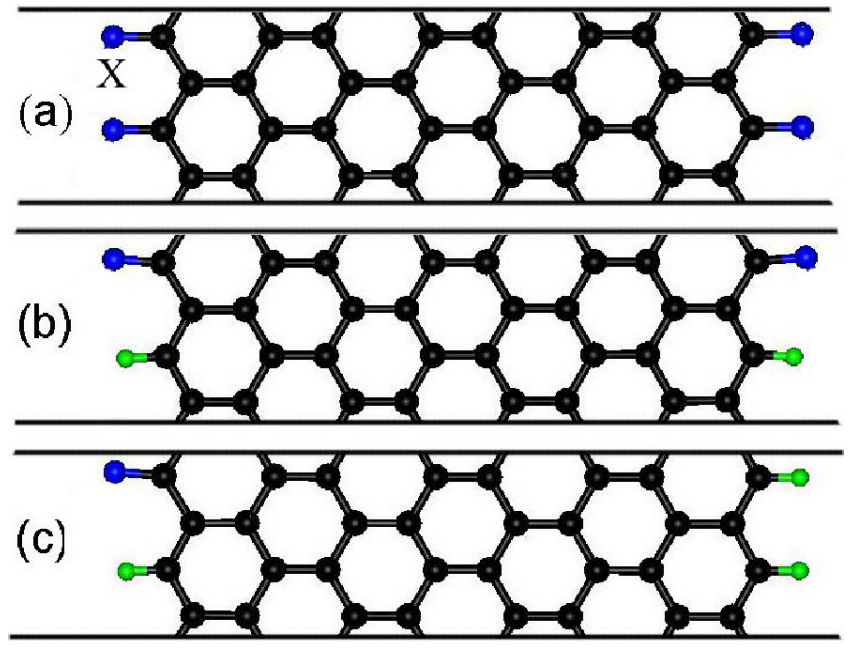

Fig. 1. Three examined zigzag graphene nanoribbons schematic. The blue, green and black balls stand for the position of the oxygen (the imine groups), hydrogen and carbon atoms, respectively

\section{RESULTS AND DISCUSSION}

At first, we calculated the electronic structures of the zigzag graphene nanoribbons which all hydrogen atoms are substituted by the oxygen atoms (thereinafter, named as 4O). The obtained results indicated that they have the spin-restricted ground states which are in agreement with the previous calculations ${ }^{8}$. Fig. 2(a) shows the band structures of the 40 zigzag graphene nanoribbons. As we discussed above, there have the edge localized electronic state which the energy states near the Fermi level are mostly localized on the edge groups. From the band structure, it is observed that there is one band cross the Fermi level twice between the $\Gamma$ and $X$ points. So these zigzag graphene nanoribbons can be used as the metallic electrodes and perhaps will present good transport properties.
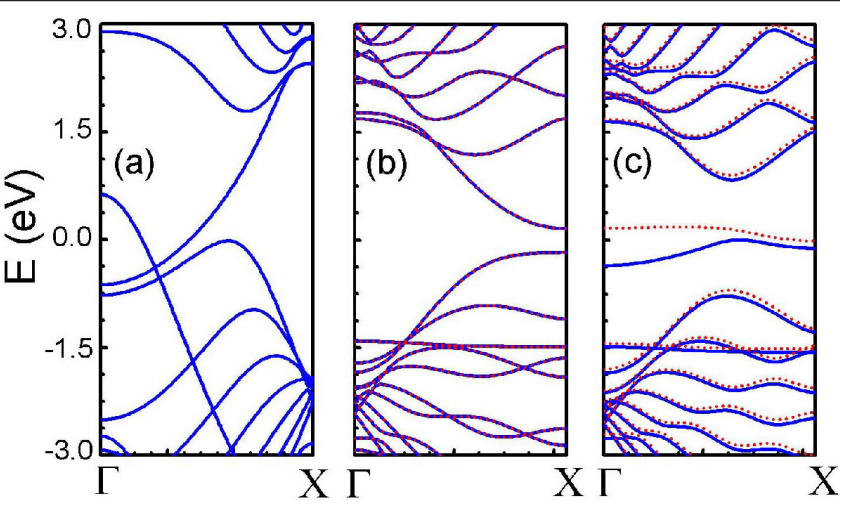

Fig. 2. Calculated band structures of $4 \mathrm{O}, 2 \mathrm{O} 2 \mathrm{H}$ and $1 \mathrm{O} 3 \mathrm{H}$ for zigzag graphene nanoribbons

It's well known that the concentration of the substituted groups is very important for tuning the electronic structure in the carbon nanotube studies. Thus, it's necessary to examine the influence of these factors in the zigzag graphene nanoribbons. Thus, the optimized geometries and the band structures of the zigzag graphene nanoribbons which have two and one oxygen atoms $(2 \mathrm{O} 2 \mathrm{H}, 1 \mathrm{O} 3 \mathrm{H})$ have been shown in Fig. 1(b), 1(c) and Fig. 2(b), 2(c), respectively. It is observed that the obtained band structures for $2 \mathrm{O} 2 \mathrm{H}$ and $1 \mathrm{O} 3 \mathrm{H}$ presented the dramatically different characteristics with the $4 \mathrm{O}$ zigzag graphene nanoribbons. As to the $2 \mathrm{O} 2 \mathrm{H}$, all bands are the spindegenerate semi-conductive states and the direct energy gap at $\mathrm{X}$ point is about $0.34(0.36) \mathrm{eV}$, respectively. However, the $1 \mathrm{O} 3 \mathrm{H}$ exhibited the obviously spin-polarized phenomena. The spin-up bands occupied the conduct bands, while the spindown states occupied the valence bands. Both the spin-up and the spin-down bands are cross the Fermi level and present the metal characteristics.

Next we turn to investigate the transport properties of the molecular junction based on the zigzag graphene nanoribbons. The schematic view of our computational model is illustrated in Fig. 3. These molecular junctions are divided into three regions: the scattering region, the left and right leads. The left and right leads are described by the 8-zigzag graphene nanoribbons with the two unit cells in the traverse direction. While the scattering region is constituted by the central molecules which have three unit cells in the traverse direction and two surface layers of the left and right leads, respectively. With the change of the surface layers, the calculated transport properties have a negligibly change, so we considered that all the screening effects are included into the contact region, which the charge-density matrix is solved self-consistently with the NEGF method under the zero bias voltage.

The calculated transmission spectra are shown in Fig. 4. Clearly, the transmission spectra are typically characterized by a sequence of the steps which have the similar properties with the carbon nanotube junctions. The height of those steps is directly related to the numbers of the sub-bands which is available for the electronic transport along the graphene nanoribbons. As for the 4O, the conducts of the junctions have the high transmission steps which are reached to $2.6 \mathrm{G}_{0}$ at the Fermi level. Below the Fermi level, the conducts become much higher with the increasing of the bands through this energy ranges. Contrast to this increasing, the conductance is only 


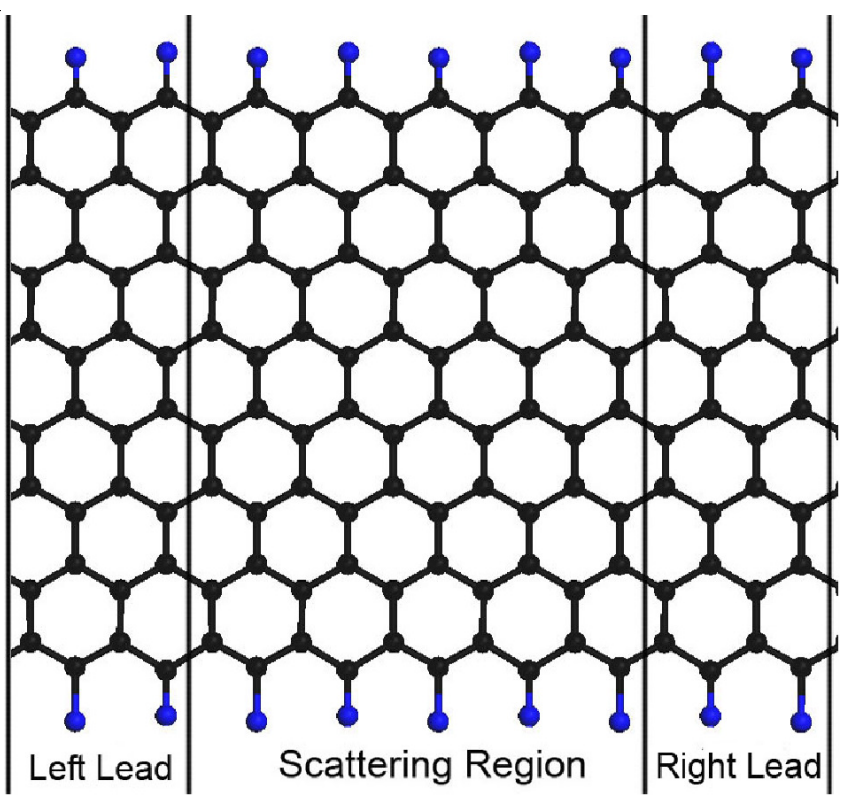

Fig. 3. Optimized two-probe geometry of 40 junctions

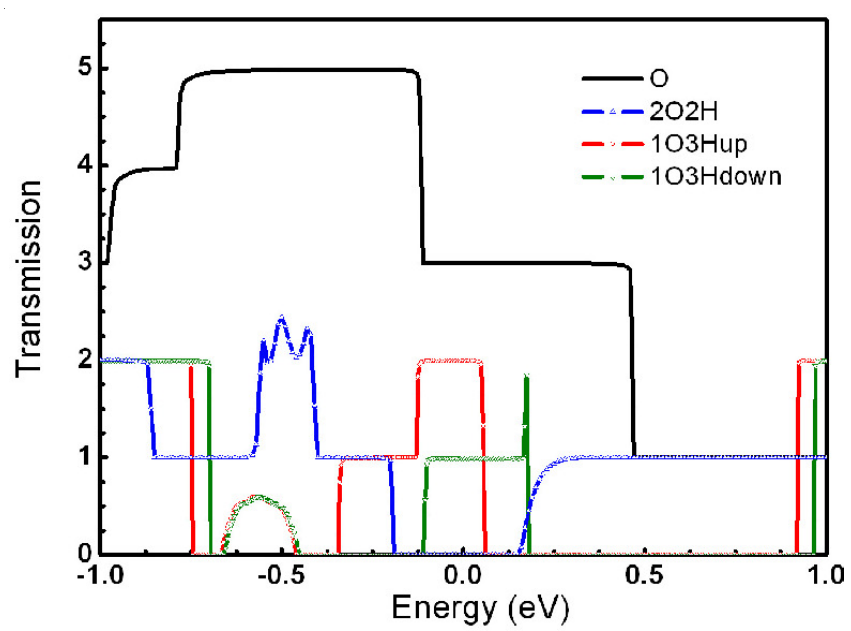

Fig. 4. Energy dependent spin-resolved transmission spectra of $4 \mathrm{O}, 2 \mathrm{O} 2 \mathrm{H}$ and $1 \mathrm{O} 3 \mathrm{H}$ junctions for zigzag graphene nanoribbons

about $1.0 \mathrm{G}_{0}$ within $[0.5,1.5] \mathrm{eV}$. These important characters predicted that the conduct ability can present the jumping increase near the Fermi level with the change from $2.6 \mathrm{G}_{0}$ at about $0.1 \mathrm{eV}$ to $5.0 \mathrm{G}_{0}$ at $0.12 \mathrm{eV}$. So we can tune the conduct switch from the high to the low states of the transport properties through the gate voltage.

As we discussed above, following the change of the concentration of the substituted groups, the molecular junctions present the distinct band structures. Thus, the transmission spectra of the $2 \mathrm{O} 2 \mathrm{H}$ indicated the distinctly characteristics with the 4O, shown in Fig. 4. Near the Fermi level, the transmission coefficients remains nearly zero within the energy region $[-0.25,0.15] \mathrm{eV}$. These phenomena are due to the vanishing of the magnetism for the hybrid of the nitrogen and carbon atoms. The localized electronic states near the Fermi level have been wiped off. So there have no any electronic states which are contributed to the conductance. While with the decrease of the substituted concentrations, the $1 \mathrm{O} 3 \mathrm{H}$ junctions demonstrated the spin-polarized transmission spectra. The transmission coefficients for the spin-up channels are remarkably larger that of the spin-down ones. For example, the calculated transmission coefficients are about $2 \mathrm{G}_{0}$ for the spin-up channels and are about twice of the spin-down ones at the Fermi level. It turns out that the conductance through the $103 \mathrm{H}$ junctions present the spin-filter effect and mainly governed by the spin-up channels.

\section{Conclusion}

In summary, we have investigated the electronic and transport properties of zigzag graphene nanoribbons with the substituted edge chemical functional groups using the first principle calculations combined with non-equilibrium Green's functions. The calculated results indicate that the concentration of the substituted groups have the obviously effect on the band structures and the conducts of the zigzag graphene nanoribbons. The transmission spectra present a series of steps when all hydrogen atoms are instead by the oxygen atoms and the height of transmission is $2.6 \mathrm{G}_{0}$ at the Fermi level. While with the decrease of the substituted concentration, the molecular junctions present the semi-conductive properties. Then we keep on decreasing the concentration until only one hydrogen atom is substituted by the oxygen atoms in the unit cells. The transmission spectra present the spin-filter effect. So it is feasible for us to controlling the transport properties using the concentration of the edge substituted groups on the zigzag graphene nanoribbons.

\section{ACKNOWLEDGEMENTS}

This work was supported by the National Natural Science Foundation of China (Grant No. 20903003).

\section{REFERENCES}

1. D. Gunlycke, J. Li, J.W. Mintmire and C.T. White, Appl. Phys. Lett., 91, 112108 (2007).

2. Y.-W. Son, M.L. Cohen and S.G. Louie, Nature, 444, 347 (2006).

3. E.J. Kan, Z.Y. Li, J.L. Yang and J.G. Hou, Appl. Phys. Lett., 91, 243116 (2007).

4. V. Hod, V. Barone, J.E. Peralta and G.E. Scuseria, Nano Lett., 7, 2295 (2007).

5. M. Brandbyge, J.L. Mozos, P. Ordejón, J. Taylor and K. Stokbro, Phys. Rev. B, 65, 165401 (2002).

6. J. Taylor, H. Guo and J. Wang, Phys. Rev. B, 63, 245407 (2001).

7. Q. Yan, B. Huang, J. Yu, F. Zheng, J. Zang, J. Wu, B.-L. Gu, F. Liu and W. Duan, Nano Lett., 7, 1469 (2007). 